\title{
Satisfacción laboral en el Servicio de Rayos X de una Institución Privada de Salud de Lima, Nivel III-2, 2015
}

\author{
Alexander H. Román Mezaํㄹ Juan P. Matzumura Kasano², Hugo Gutiérrez Crespo ${ }^{3}$
}

\section{RESUMEN}

Objetivo: Describir la satisfacción laboral en el Servicio de Rayos X de una institución privada de salud de Lima, nivel III-2, en el año 2015.

Material y Métodos: La presente investigación es de tipo cuantitativo, descriptivo, prospectivo y de corte transversal. La población estuvo constituida en total por 22 personas, y la muestra estuvo conformada por todos los elementos de la población. La unidad de análisis fue el Tecnólogo Médico de Radiología. La satisfacción laboral se evaluó mediante el Cuestionario de Satisfacción Laboral S10/12 de J.L. Meliá y J.M. Peiró.

Resultados: De la población total, $77.3 \%$ de personas se encontraba contratada a plazo fijo y un $40.9 \%$ venía trabajando de 12 meses a más.El 86.4\% de los participantes manifestaron estar satisfechos laboralmente. Un $65.9 \%$ mostró satisfacción con la dimensión prestaciones recibidas, y 10.2\% mostró satisfacción indiferente con la dimensión ambiente físico.

Conclusión: La satisfacción laboral fue alta en el servicio, pero en el análisis, se definió que es el ambiente físico en donde la institución debe mejorar, en comparación a las prestaciones recibidas y la supervisión. (Horiz Med 2015; 15(3): 20-25)

Palabras clave: Satisfacción laboral, servicio de rayos x, condiciones laborales. (Fuente: DeCS BIREME).

Job satisfaction in the X-Ray Service of a Level III-2 Private Health Institution in Lima, 2015

\section{ABSTRACT}

Objective: To describe job satisfaction in the X-Ray Department of a level III-2 private health institution in Lima, in 2015. Material and Methods: The present study is quantitative, descriptive, prospective and cross-sectional. The population was of 22 people, the sample included everyone in the population. The unit of analysis was the Medical Technologists of Radiology. The job satisfaction was evaluated by the "Job Satisfaction Questionnaire S10 / 12" by Melia JL and Peiró JM.

Results: Of the total population, $77.3 \%$ were hired on a fixed term and $40.9 \%$ had been working for 12 months or more. $86.4 \%$ of participants were occupationally satisfied. $65.9 \%$ expressed satisfaction with the services received dimension, and $10.2 \%$ had an indifferent satisfaction with the physical environment dimension.

Conclusion: J ob satisfaction was high in the department but in the analysis, it was determined that the physical environment is where the institution must improve, compared to the benefits received and supervision. (Horiz Med 2015; 15(3): 20-25)

Key words: Job satisfaction, x-rays department, working conditions (Source: MeSH NLM).

1 Licenciado en Tecnología Médica de Radiología. Centro de Diagnóstico por Imágenes de Clínica Internacional - Sede San Borja. Miembrode la Sociedad Peruana de Tecnólogos en Radiología, Tomografía Computada y Resonancia Magnética (SOPTRATOREM).

2 Médico Ginecólogo Obstetra. Doctor en Medicina. Docente de Postgrado de la Facultad de Medicina de la UNMSM. Miembro del Consejo Directivo del Sistema de Certificación y Recertificación del Colegio Médico del Perú. Presidente de la Federación Latinoamericana de Sociedades de Climaterio y Menopausia (FLASCYM).

3 Obstetra. Magíster en Docencia e Investigación en Salud. Coordinador Académico de la Sociedad Peruana de Obstetricia y Ginecología (SPOG). Lima-Perú. 


\section{INTRODUCCIÓN}

La satisfacción laboral, también denominada como satisfacción del usuario interno, no posee una definición única, ha sido estudiada por varios especialistas y ha tenido varias denominaciones: actitud del empleado, moral laboral, satisfacción en el trabajo; pero en general, es una actitud o conjunto de actitudes desarrolladas por la persona hacia su situación de trabajo, las cuales pueden ir referidas hacia el trabajo en general o facetas específicas del mismo (1).

En el marco de la psicosociología, la satisfacción laboral se ha definido de muchas formas, las que confluyen en que representa el grado de bienestar que experimenta el trabajador con motivo de su trabajo, y constituye uno de los principales indicadores de la calidad de vida laboral, por lo que tanto la satisfacción como insatisfacción se convierten en agentes motivadores del trabajo, los cuales generarán actitudes positivas o contrarias respectivamente (2).

Debe entenderse como "una función de la satisfacción en los diferentes aspectos del trabajo" (modelo aditivo), o bien ser percibida como "la diferencia existente entre el grado en que se satisfacen o colman las necesidades de las personas (realidad) y el grado en que deberían satisfacerse (idealmente)"; de esta forma constituye un concepto íntimamente relacionado, pero no equivalente, a la motivación, clima y comunicación organizacionales (3).

Dado que la satisfacción laboral se refiere a las actitudes del empleado hacia su trabajo real en comparación con su trabajo ideal, y la relación de estas con su vida en general, influyen factores del propio empleado como: necesidades, valores, rasgos personales y habilidades, los cuales se verán afectados en ocasiones por aspectos del empleo como: condiciones favorables, identidad y significación de la tarea, supervisión, autonomía, recompensas, seguridad en el empleo y promociones, entre otros (4).

Al ser una actitud afectiva, tiene una fuerte relación con su propio medio social, por lo que se vuelve dinámica; es decir, que puede disminuir considerablemente hasta con un hecho aislado, pero que puede fortalecerse cuando se aplican buenas prácticas en materia de recursos humanos, como los programas organizacionales orientados a dar mayor participación y más información (5).

Por haber sido estudiada por varios investigadores, se han definido técnicas válidas y fiables que permiten realizar su medición, tanto de forma global, como atendiendo a dimensiones específicas de la misma, y obtener así información sobre grupos particulares de empleados sobre la satisfacción, de cara a prevenir y modificar en lo posible aquellas actitudes negativas que puedan existir (1).

Por todo ello, es evidente que al medir la satisfacción se comprueba la calidad de la organización y sus servicios. Por lo tanto, el grado de satisfacción se verá reflejado en la calidad de las prestaciones, con lo que es importante que las instituciones se preocupen por buscar que los empleados posean una alta satisfacción porque les permitirá ser capaces de adaptarse a las exigencias laborales actuales; esto, en una institución de salud, se dará tanto en el aumento de las atenciones a realizar como la tecnología a utilizar (6).

En ese sentido, una de las áreas más influenciadas por estas exigencias es el Servicio de Rayos X, el cual; gracias a los enormes avances en bioinformática, ha establecido protocolos electrónicos de trabajo que permiten adquirir las imágenes, archivarlas y distribuirlas, permitiendo obtener el diagnóstico inclusive el mismo día y así poder iniciarse el tratamiento inmediatamente.

Lo anterior, ha generado una predilección por parte del médico solicitante y el paciente como uno de los métodos favoritos para el diagnóstico (7).

Por tal razón, es importante que haya una eficiencia en la realización de estos exámenes, de manera que esta innovación tecnológica sí ayude a determinar un diagnóstico precoz, una decisión clínica certera, y/ o una elección del tratamiento más acorde para cada paciente, lo que representará un menor costo del proceso global de atención y a la vez que se dé un mayor número de exámenes a realizar, lo cual 
generaría un sustento económico para la institución y la sostenibilidad del servicio, en especial en las instituciones privadas (8).

Sin embargo, en el país no existen estudios sobre satisfacción laboral del personal de este servicio, tanto en la institución pública como en la privada, siendo en esta última más relevante por lo que representa económicamente.

Esto sorprende, considerando que la calidad en cualquier servicio radiológico no solo pasa por preocuparse por los usuarios externos (pacientes a los cuales se les realiza un examen y los médicos solicitantes de las pruebas), sino también en su usuario interno (empleados); es decir, satisfacer las expectativas de todos los grupos de interés. Así, es evidente que al medir la satisfacción laboral se comprueba la calidad de la organización y sus servicios internos, satisfacción que se verá reflejada en la calidad de los servicios prestados (6).

El objetivo de este estudio fue describir la satisfacción laboral en el Servicio de Rayos X de una institución privada de salud de Lima, nivel III-2, en el año 2015.

\section{MATERIAL Y MÉTODOS}

Estudio de tipo cuantitativo, descriptivo, prospectivo y de corte transversal. La población estuvo constituida en total por 22 personas. La muestra fue no probabilística y estuvo conformada por todos los elementos de la población. La unidad de análisis fue el Tecnólogo Médico de Radiología. Para la recolección de información, se seleccionó como técnica el cuestionario autoadministrado $y$ el instrumento utilizado fue el "Cuestionario de Satisfacción Laboral S10/12" de J.L. Meliá y J.M. Peiró (versión en español) para obtener un diagnóstico general y que contiene la descripción de tres dimensiones:

1 satisfacción con la supervisión

2 satisfacción con el ambiente físico y

3 satisfacción con las prestaciones recibidas; constituida por 12 preguntas respondidas mediante una escala de Likert de 7 puntuaciones, que va de 1 a 7 (muy insatisfecho, bastante insatisfecho, algo insatisfecho, indiferente, algo satisfecho, bastante satisfecho, y muy satisfecho).

El cuestionario presenta un nivel de fiabilidad y validez apreciables: Coeficiente a de Cronbach de 0,88 y los factores oscilan entre 0,72 (factor II), y 0,89 (factor I), pasando por 0,74 (factor III) (1).

\section{RESULTADOS}

La edad de las personas entrevistadas varió de 24 a 42 años, alcanzando una mediana de 30 años. El $59.1 \%$ fue de sexo femenino, mientras que un $40.9 \%$ fue de sexo masculino. El $77.3 \%$ tenía contrato a plazo fijo y los demás tenían: contrato por inicio de actividades (13.6\%) y contrato por tiempo indeterminado (9.1\%). Del total, $40.9 \%$ tenían un tiempo de trabajo mayor a 12 meses, $40.9 \%$ menor a 12 meses y $18.2 \%$ menor a 6 meses.

En general, el total de los entrevistados refirieron estar satisfechos con la supervisión. Aunque, de acuerdo a los ítems relacionados a la dimensión de la supervisión, el $63.6 \%$ de los entrevistados refirieron estar satisfechos en cuanto a las relaciones personales con sus superiores, el $54.5 \%$ refirió estar satisfecho con la supervisión que le ejercen, el $45.5 \%$ refirió estar satisfecho en cuanto a la proximidad y frecuencia con que es supervisado, el $59.1 \%$ refirió estar satisfecho en relación a la forma en que sus supervisores juzgan sus tareas, el $54.5 \%$ refirió estar satisfecho en cuando a la igualdad y justicia de trabajo que recibe de su empresa, y el $59.1 \%$ de ellos refirieron estar satisfechos con el apoyo que recibe se sus superiores. (Tabla 1)

Asimismo, el total de los entrevistados refirieron estar satisfechos con el ambiente físico (muy satisfecho $18.2 \%$, bastante satisfecho $50.0 \%$ ), aunque en menor porcentaje que la dimensión supervisión. De acuerdo a sus ítems relacionados en esta dimensión, el $40.9 \%$ de los entrevistados refirieron estar satisfechos en cuanto al logro de sus objetivos, metas y tasas de producción, el $59.1 \%$ 
refirió estar satisfecho en relación a la limpieza, higiene y seguridad en su lugar de trabajo, el $40.9 \%$ refirió estar satisfecho en cuanto al entorno físico y el espacio que dispone en su lugar de trabajo, y el $59.1 \%$ refirió estar satisfecho en relación a la temperatura del ambiente de trabajo. (Tabla 1)
En la dimensión de las prestaciones recibidas, el $81.8 \%$ de los entrevistados refirieron estar satisfechos en relación al grado en que su empresa cumple con el convenio, disposiciones y leyes laborales.

Tabla 1. Frecuencias para cada ítem del Cuestionario $\mathrm{S} 10 / 12$

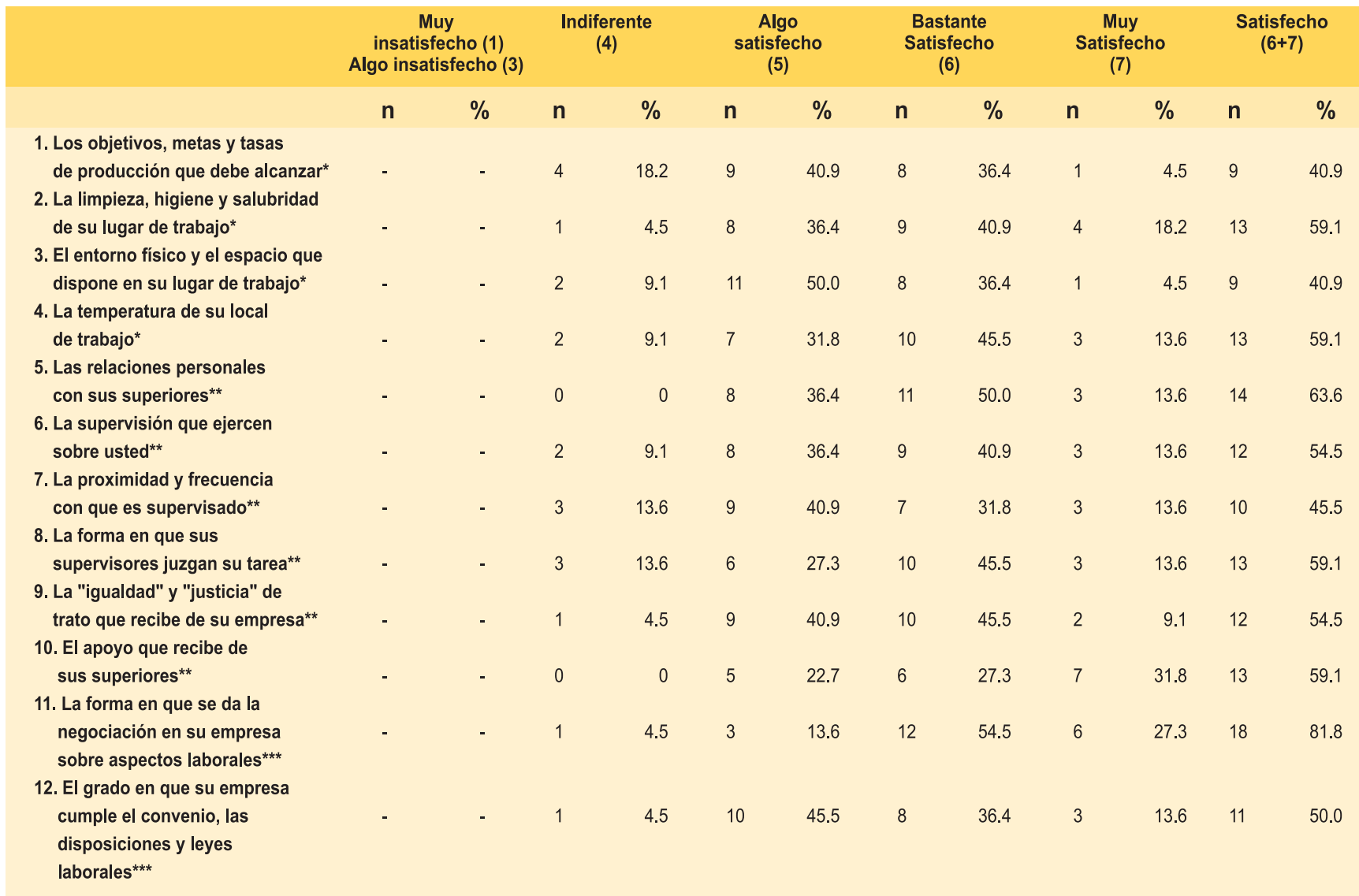

* $\quad$ Preguntas de la dimensión ambiente físico.

* Preguntas de la dimensión supervisión.

*** Preguntas de la dimensión prestaciones recibidas.

Sin embargo, solo el 50\% de entrevistados refirieron estar satisfechos con la forma en que se da la negociación en la empresa sobre aspectos laborales. (Tabla 1)

Realizando un análisis comparativo de las tres dimensiones medidas por el cuestionario se observa que existe una mayor satisfacción con las prestaciones recibidas (65.9\%). Asimismo, en la dimensión del ambiente físico es donde existe mayor satisfacción indiferente (10.2\%); es decir existe un menor grado de satisfacción. (Figura 1)

En síntesis, $18.2 \%$ y $68.2 \%$ de los entrevistados refirieron estar muy satisfechos y bastante satisfechos, respectivamente; es decir, el $86.4 \%$ refirió estar satisfecho laboralmente, mientras que un 13.6\% refirió estar algo satisfecho. (Figura 2). 


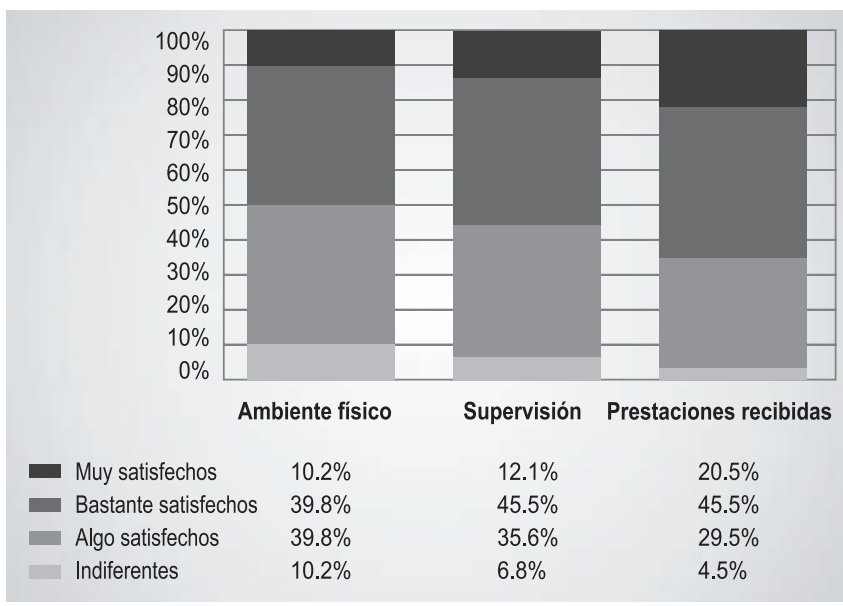

Figura 1. Satisfacción laboral por dimensiones involucradas

\section{DISCUSIÓN}

Los resultados del estudio en su mayoría de ítems demostraron que las personas están satisfechas. Aunque no existen estudios de satisfacción laboral en un Servicio de Rayos $X$ ni en algún otro tipo de servicio radiológico, existen investigaciones sobre satisfacción de usuarios internos en salud, los cuales llegaron a conclusiones similares respecto a la satisfacción laboral.

Cabe considerar que los estudios referenciales no valoran exactamente los mismos criterios considerados por el instrumento de medición utilizado.

Comparando el presente estudio con el realizado por Vargas (9) en los Servicios de Salud del Patronato Provincial de Pichincha (Ecuador) y Carrasco y Col (10) en el Centro de Salud No. 1 de la ciudad de Durango (México), donde el objetivo en general fué conocer la satisfacción del usuario interno y analizar los factores asociados, se observó un alto porcentaje de satisfacción laboral en ambos casos: $90 \%$ y $71 \%$ respectivamente, coincidiendo con los resultados de este estudio, donde se obtuvo un $86.4 \%$ de personas satisfechas.

El aspecto del ambiente físico, denominado en cada referencia como instalaciones y condiciones físicas, fue uno de los factores donde se presentó mayor insatisfacción en comparación a los demás:

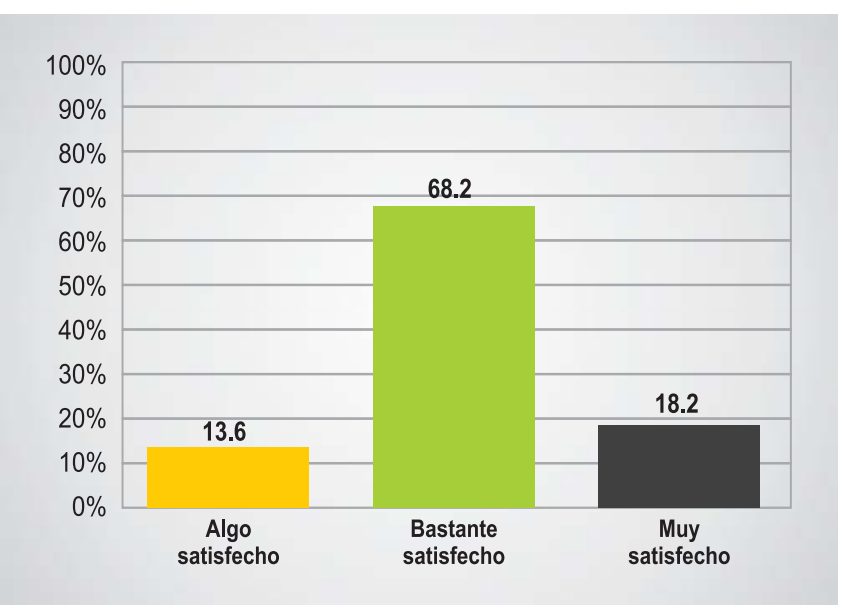

Figura 2. Satisfacción laboral por dimensiones involucradas

$31 \%$ y $65 \%$ respectivamente, de forma similar a este estudio donde fue la dimensión con menor satisfacción de las tres abordadas, con un $10.2 \%$ de personas con satisfacción indiferente (mayor al $6.8 \%$ de la dimensión supervisión y $4.5 \%$ de la dimensión prestaciones recibidas).

Esto demostraría que es un aspecto en el cual las instituciones en Latinoamérica tienden a no darle mucha importancia, pero que sí debería mejorarse para brindarle una mayor comodidad al personal para la realización de su trabajo y con ello elevar el nivel de satisfacción general de los trabajadores.

Asimismo, en investigaciones realizadas sobre satisfacción del usuario interno en nuestro país por Apaza (11) en el Puesto de Salud SASBI de Villa El Salvador, Kcomt (12) en el Servicio de Emergencia del Hospital Nacional Sergio E. Bernales, y Díaz (13) en el Policlínico de La Victoria (Chiclayo), se encontró porcentajes aceptables de satisfacción laboral: $88.9 \%, 50 \%$ y $75 \%$ respectivamente, que también coincidieron con los resultados de este estudio, donde se obtuvo un $86.4 \%$ de personas satisfechas.

El aspecto de las prestaciones recibidas, denominado en las dos primeras referencias $(11,12)$ como incentivos y en la otra $(13)$ como beneficios laborales, se encontró como uno de los factores con menor grado de satisfacción: $22.2 \%$ $15.6 \%$ y $18 \%$ respectivamente, lo cual también fue contrario a los resultados de este estudio donde ha sido la dimensión con mayor satisfacción: $65.9 \%$ de 
personas satisfechas (satisfacción mayor al 57.6\% de la dimensión supervisión y $50 \%$ de la dimensión ambiente físico).

Esta situación demuestra cómo se manejan muchas veces las condiciones laborales y prioridades dentro de los servicios de salud en el Perú: mientras que en el sector público están más enfocados en que exista una infraestructura física adecuada y espaciosa para realizar las labores y se descuida lo que se le va a brindar al personal por su labor (prestaciones recibidas), en el sector privado se da la situación contraria: se maneja de mejor forma las prestaciones que recibe el personal, pero no siempre se cuenta con ambientes acordes para trabajar.

En conclusión, la mayoría de personas se encontraban satisfechas laboralmente, teniendo la dimensión de supervisión el mayor grado de personas satisfechas. Con menor grado de satisfacción fue la del ambiente físico.

\section{Fuentes de financiamiento}

Este artículo ha sido financiado por el autor.

\section{Conflicto de interés}

Los autores declaran no tener conflictos de interés.

\section{Correspondencia:}

Alexander H. Román Meza

Correo: alexrom2490@gmail.com

Dirección: Cl. Tarata 493. La Perla - Callao.

Teléfono: 949071089 - 4202208

\section{REFERENCIAS BIBLIOGRÁFICAS}

1. Chiang M, Martín MJ, Núñez A. Relaciones entre el clima organizacional y la satisfacción laboral. Madrid: Universidad Pontificia Comillas; 2010.

2. Nieto C. Los efectos del trabajo con personas mayores dependientes institucionalizadas. Madrid: Editorial Dykinson; 2014.

3. Gan F, Triginé J. Clima laboral. $15^{\mathrm{a}}$ ed. Madrid: Ediciones Díaz de Santos; 2012.

4. Robbins S, Judge T, Enríquez J. Comportamiento organizacional. $15^{\mathrm{a}}$ ed. México: Pearson; 2013.

5. Alles M. Conciliar vida profesional y personal. Buenos Aires: Ediciones Granica; 2010.

6. Temes J, Mengibar M. Gestión Hospitalaria. $5^{\mathrm{a}}$ ed. Madrid: McGrawHill - Interamericana; 2011.

7. McCall I. The future role of radiology in healthcare. Insights Imaging. 2010; 1(1):2-11

8. Martí-Bonmatí L. Retos y desafíos tecnológicos de la Radiología. Gest y Eval Cost Sanit. 2013; 14(3):379-386.

9. Vargas D. Estudio de satisfacción del Usuario Externo e Interno en los servicios de salud del Patronato Provincial de Pichincha [tesis de maestría]. Quito: Colegio de Postgrados, Universidad San Francisco de Quito; 2013.

10. Carrasco R, Barraza A, Arreola M. Satisfacción laboral en personal del centro de salud No. 1 de la ciudad de Durango, México. Psico Pedia Hoy. [revista en Internet] 2013 [consultado 30 marzo 2015]; 15(2). Disponible en: http:// psicopediahoy.com/satisfaccionlaboral-centro-de-salud

11. Apaza R. Nivel de satisfacción del usuario interno del Puesto de Salud SASBI de Villa El Salvador [tesis]. Lima: Facultad de Medicina, Universidad Nacional Mayor de San Marcos; 2014.

12. Kcomt R. Relación entre la satisfacción del usuario interno y externo del Servicio de Emergencia del Hospital Nacional Sergio E. Bernales de Collique [tesis de especialidad]. Lima: Facultad de Medicina, Universidad Nacional Mayor de San Marcos; 2013.

13. Díaz S. Satisfacción laboral y Síndrome de Burnout entre el personal de un Policlínico y en el de una Comunidad Local de Administración de Salud, Distrito La Victoria, Chiclayo - 2012 [tesis]. Chiclayo: Facultad de Psicología, Universidad Católica Santo Toribio De Mogrovejo; 2013.
Recibido: 21 de Junio de 2015 Aprobado: 06 de Agosto de 2015 